\title{
Diagnóstico del desarrollo de Pinus massoniana y Pinus brutia establecidos en el arboreto de la Universidad Austral de Chile, Valdivia
}

\author{
Diagnosis for the development of Pinus massoniana and Pinus brutia established \\ in the arboretum at Universidad Austral de Chile, Valdivia
}

\author{
Víctor Gerding ${ }^{1 *}$, Edith Geldres ${ }^{2}$, Jorge A. Moya ${ }^{1}$ \\ * Autor de correspondencia: ${ }^{1}$ Universidad Austral de Chile, Instituto de Silvicultura, Valdivia, Chile, vgerding@uach.cl \\ ${ }^{2}$ Universidad de Los Lagos, Departamento de Ciencia y Tecnología Forestal, Osorno, Chile.
}

\begin{abstract}
SUMMARY
A diagnosis of the site and of the development of 4-year-old P. massoniana and P. brutia established in 1999 in the arboretum at Universidad Austral de Chile in Valdivia (3948' S and 73ำ14' W) was made. In this site, the climate is west-coastal type with Mediterranean influence, presenting an annual precipitation of $2,300 \mathrm{~mm}$ and an mean annual temperature of $12{ }^{\circ} \mathrm{C}$, without occurrence of dry periods. The soil is composed of recent volcanic ashes deposited on metamorphic rock; deep, silty loam and without structural or drainage boundaries for rooting; rich in organic matter and total nitrogen, showing low levels of removable bases, a very low presence of phosphorous, strongly acid $\mathrm{pH}$ and a high saturation of exchangeable aluminum.

Both species showed $100 \%$ survival and were affected by the competition of grass species during the first years. Pinus massoniana presented an average height of $3 \mathrm{~m}$ and a crown diameter of $1.5 \mathrm{~m}$; its sanitary status is good, without any evidence of biotic damage; the crowns still have their foliage intact and its nutritional condition is adequate. This species evidences good adaptation to the site. Pinus brutia reached an average height of $1.9 \mathrm{~m}$ and a crown diameter of $1.0 \mathrm{~m}$; its sanitary status is deficient due to the attack of the woolly pine aphid (Pineus borneri, Adelgidae family) and of red band disease (Dothistroma septospora); the crowns have lost their old foliage, keeping that from the last two periods. Its nutritional condition, however, is good. This species evidences climatic incompatibility and high sensitivity to some agents causing biotic damage.
\end{abstract}

Key words: Pinus massoniana, Pinus brutia, diagnosis, site, arboretum.

\section{RESUMEN}

Se realizó un diagnóstico del sitio y del desarrollo de $P$. massoniana y $P$. brutia de 4 años establecidos en 1999 en el arboreto

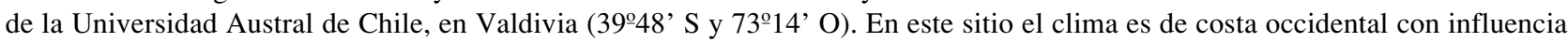
mediterránea, con precipitaciones anuales de $2.300 \mathrm{~mm}$ y temperatura media anual de $12{ }^{\circ} \mathrm{C}$, sin ocurrencia de períodos secos. El suelo es de cenizas volcánicas recientes depositadas sobre esquistos metamórficos; profundo, franco limoso y sin limitaciones estructurales ni de drenaje para el arraigamiento; rico en materia orgánica y nitrógeno total, presenta bajos niveles de bases extraíbles, muy baja oferta de fósforo disponible, $\mathrm{pH}$ fuertemente ácido y alta saturación de aluminio intercambiable.

Ambas especies presentaron $100 \%$ de sobrevivencia y estuvieron afectadas por competencia de especies herbáceas en los primeros años. Pinus massoniana presentó una altura promedio de $3 \mathrm{~m}$ y diámetro de copa de 1,5 m; su estado sanitario es bueno, sin evidencias de daños bióticos; las copas mantienen su follaje completo y su estado nutritivo es adecuado. Esta especie manifiesta una buena adaptación al sitio. Pinus brutia alcanzó una altura promedio de 1,9 m y diámetro de copa de 1,0 m; su estado sanitario es deficiente por el ataque de pulgón lanígero (Pineus borneri, familia Adelgidae) y del hongo de la banda roja (Dothistroma septospora); sus copas han perdido el follaje antiguo, conservado el de los últimos dos períodos; sin embargo, su estado nutritivo es bueno. Esta especie manifiesta incompatibilidad climática y alta susceptibilidad ante algunos agentes de daño bióticos.

Palabras clave: Pinus massoniana, Pinus brutia, diagnóstico, sitio, arboreto.

\section{INTRODUCCIÓN}

Para el desarrollo de programas de introducción de especies forestales o para incrementar el conocimiento sobre ellas se puede contar con información proveniente de diversas fuentes experimentales como los arboretos.
En el arboreto de la Universidad Austral de Chile se cultivan unas 500 especies forestales, la mayoría exóticas (Huber 1995). Entre ellas se encuentran establecidos, desde 1999, el pino rojo chino (Pinus massoniana Lamb.) y el pino afgano ( $P$. brutia Ten. var. eldarica (Medw.) Silba). Un diagnóstico del sitio y de estas plantaciones 
experimentales permite evaluar el desarrollo en la etapa juvenil de estas especies en su nuevo hábitat. Con ello se pueden detectar tempranamente las limitaciones y potencialidades que presentan estas especies en sitios como los del arboreto y se puede proyectar su crecimiento. Igualmente, se puede obtener información relevante sobre el comportamiento de la especie introducida con respecto a su posible efecto invasor hacia otros ecosistemas donde pudiese producir efectos no deseados.

Con respecto al sitio, Gerding y Schlatter (1995), Schlatter y Gerding (1995) y Schlatter et al. (1995) sintetizan los principales factores y su ponderación para la producción vegetal, particularmente de bosques. La consideración principal está en el clima, ya que la compatibilidad climática es esencial para el éxito de una introducción de especie vegetal. Se evalúa desde el macroclima hasta las variaciones microclimáticas provocadas, por ejemplo, por cambios topográficos como exposición y elevación. En segundo lugar debe ponderarse el suelo en sus aspectos estructurales que determinan el espacio arraigable y los regímenes de aire y agua. En tercer lugar, el régimen de elementos nutritivos juega un papel importante en el éxito de la producción forestal. Sin embargo, este último factor juega un rol significativo sólo si los anteriores factores otorgan condiciones adecuadas para el establecimiento y desarrollo de una cubierta vegetal determinada.

El objetivo de este trabajo fue evaluar el actual estado de desarrollo de sendas plantaciones jóvenes de $P$. massoniana y $P$. brutia establecidas en el arboreto de la Universidad Austral de Chile, a través de un diagnóstico que integra el sitio y el estado actual de las plantaciones.

\section{CARACTERÍSTICAS DE LAS ESPECIES Y DE LOS SITIOS DE ORIGEN}

Pinus massoniana crece desde el nivel del mar hasta los $2.000 \mathrm{~m}$ s.n.m., en una extensa zona del sureste de la República Popular China y Vietnam (Vidaković 1991). El clima de la zona se caracteriza por presentar inviernos suaves, con temperaturas por sobre $\operatorname{los} 0{ }^{\circ} \mathrm{C}$ hasta los 15 ${ }^{\circ} \mathrm{C}$, y para los meses de verano fluctuaciones entre los 20 y los $30{ }^{\circ} \mathrm{C}$. Las precipitaciones están homogéneamente repartidas durante el año, siendo de 50 a $200 \mathrm{~mm}$ en los meses de invierno, y de 100 a $300 \mathrm{~mm}$ para la estación de crecimiento. El hábitat de $P$. massoniana es principalmente rústico, debido al amplio rango de distribución, creciendo en variados tipos de suelos, situación que es altamente compatible con los suelos ácidos de la región del estudio. Sin embargo, sus expectativas de crecimiento son mejores en suelos profundos, porque poseen un sistema radicular que profundiza. En su zona de origen $P$. massoniana es un árbol que crece entre 18 y $45 \mathrm{~m}$ de altura, pudiendo alcanzar $150 \mathrm{~cm}$ de DAP. Es una especie muy intolerante, de alta exigencia de agua y se desa- rrolla con altos contenidos de humedad del aire; de crecimiento rápido en sus primeros estadios de desarrollo, para después ser más lento.

En su zona de origen, $P$. brutia var. eldarica crece por sobre los 1.000 metros de altitud, situación que se ve reflejada en las tasas moderadas a lentas en su crecimiento y alta longevidad (Vidakovic 1991). Esta especie se desarrolla principalmente en zonas semiáridas de Asia Central con veranos secos $(<25 \mathrm{~mm}$ de precipitación) y extremadamente calurosos $\left(25\right.$ a $\left.35^{\circ} \mathrm{C}\right)$, creciendo a luz plena en estado natural. Las escasas precipitaciones se concentran principalmente como nieve en los meses más fríos (precipitación: 25 a $50 \mathrm{~mm}$; temperaturas: -5 a 15 $\left.{ }^{\circ} \mathrm{C}\right)$. Producto del derretimiento de la nieve se obtiene una importante cuota de agua aprovechable, lo cual hace que el período de abundancia de humedad en el suelo esté limitado a un tiempo muy breve. Por su carácter continental el período de crecimiento está restringido a unos pocos meses durante el año. Pinus brutia crece en áreas extremadamente secas, donde el suelo es en general poco evolucionado, presentando un $\mathrm{pH}$ con tendencia a la alcalinidad. Sin embargo, se adapta a cualquier tipo de suelo, sean estos ácidos o alcalinos, arenosos o arcillosos. Pero, debido a la escasa pluviometría, necesita suelos profundos que le garanticen un suministro de humedad suficiente para los meses de déficit hídrico.

Pinus brutia se desarrolla como árbol mediano a pequeño de 9-15 m de altura en estado natural, de forma simétrica y tiende a redondearse con la edad. Es una especie intolerante, de baja exigencia de agua y se desarrolla con bajo contenido de humedad del aire. Crece moderadamente lento cuando es joven y vigoroso cuando sobrepasa los 50 años.

\section{MÉTODOS}

Clima. El área de estudio está ubicada en el arboreto perteneciente a la Universidad Austral de Chile (Isla Teja, ciudad de Valdivia), con latitud 39\%48' S y longitud 731' $\mathrm{O}$, en terrenos bajos, de lomajes fuertemente ondulados con cotas que varían hasta los 60 metros de elevación (Schlegel 1985). El clima está clasificado como de costa occidental con influencia mediterránea (Cfsb) (Fuenzalida 1965). Según Schlatter et al. (1995), el régimen de humedad anual se caracteriza por abundantes precipitaciones que sobrepasan los $2.000 \mathrm{~mm}$ anuales. Rara vez hay períodos secos. Esta situación representa para la vegetación altos excedentes de humedad para el normal desarrollo de una abundante vegetación nativa. La humedad relativa del aire es generalmente alta, con un rango de promedios mensuales de 67 a $89 \%$. El régimen térmico anual presenta sólo 30 a 60 días al año con heladas y 200 a 300 días al año libres de heladas. Las temperaturas mínimas se presentan en 2 a 3 meses del año (invierno), alcanzando $4{ }^{\circ} \mathrm{C}$ bajo cero. La limitante principal para el 
normal desarrollo de las especies vegetales en esta zona es la ocurrencia de heladas tempranas y los efectos del viento, especialmente en áreas con elevaciones mayores. Los efectos de un eventual período seco o de un déficit hídrico y las temperaturas extremas son amortiguados significativamente por su cercanía al mar. Los datos consignados por Huber (1970) y los de años recientes (período 1995-2001) ${ }^{1}$ mostraron la misma tendencia: precipitación anual de 1.394 a 2.937 mm, 36 a 77 días con heladas por año y temperaturas de 11,7 a $12,8{ }^{\circ} \mathrm{C}$ de media anual, 5,4 a $10,7^{\circ} \mathrm{C}$ de media mensual en invierno y 13,7 a 19,0 ${ }^{\circ} \mathrm{C}$ de media mensual en verano.

Suelos. La geomorfología del lugar corresponde a planos depositacionales remanentes y terrazas aluviales en posiciones bajas e intermedias. La topografía es fuertemente ondulada a ligeramente quebrada, con una pendiente promedio del $10 \%$ y con un drenaje externo rápido. El área de estudio se encuentra aproximadamente a $50 \mathrm{~m}$ s.n.m, con exposición noroeste, en una ladera alta de forma y contorno convexos. Los suelos se formaron de cenizas volcánicas con dominio de la serie Correltúe (Andic Palehumult), originada de cenizas volcánicas antiguas, con influencia en la superficie de cenizas volcánicas modernas de la serie Valdivia (Duric Hapludand) (Schlatter et al. 1995, CIREN 1999, Düsterhöft 2003).

El suelo se caracterizó, según Schlatter et al. (2003), a través de un reconocimiento mediante bastón pedológico y descripción del perfil. Se analizó químicamente ${ }^{2}$ una muestra mezcla de suelo de $0-20 \mathrm{~cm}$ de profundidad: $\mathrm{pH}$ en agua y en $\mathrm{KCl} 0,1 \mathrm{~N}$ (relación suelo: solución de $1: 2,5)$, carbono total por oxidación con dicromato de potasio y ácido sulfúrico (determinación colorimétrica), nitrógeno total (Kjeldahl, determinación colorimétrica); fracción extraíble de sodio, potasio, calcio, magnesio, aluminio, hierro, cobre, zinc y manganeso (acetato de amonio a $\mathrm{pH}$ 4,8-DTPA), fósforo disponible (Olsen), azufre disponible (S-sulfato en extracto de fosfato de calcio), boro en extracto $\mathrm{CaCl}_{2}, 0,15 \%$ a ebullición, aluminio intercambiable $(\mathrm{KCl} 1 \mathrm{M})$. En cada horizonte se determinó la densidad aparente (método del cilindro, tres repeticiones) y la resistencia a la penetración con un penetrómetro de punta circular plana (10 repeticiones).

El perfil de suelo presentó horizontes A1 (0-6 cm), A2 $(6-26 \mathrm{~cm}), \mathrm{AB}(26-33 \mathrm{~cm}), \mathrm{BA}(33-65 \mathrm{~cm})$ y B $(65-$ $>100 \mathrm{~cm}$ ), con una profundidad arraigable de más de 100 $\mathrm{cm}$ y textura franca limosa en todo el perfil. El color mostró una variación típica debido a la influencia de la materia orgánica en los primeros horizontes, variando desde pardo oscuro (7,5YR3/2) a pardo rojizo oscuro en

Estación climática Teja (39²48' S, 73ำ14' O, 9 m s.n.m.)

Todos los análisis se realizaron en el Laboratorio de Nutrición y Suelos Forestales, Facultad de Ciencias Forestales, Universidad Austral de Chile. profundidad (5YR4/3); también influyó en el color de los horizontes $\mathrm{A} 2$ y $\mathrm{AB}$, principalmente, el efecto del fuego que se utilizó antiguamente como práctica habitual de manejo, lo que provocó la presencia de moteados de coloración rojiza. La estructura del suelo varió de granular y subpoliédrica en la superficie a masiva en profundidad. La consistencia fue homogénea en todo el perfil, variando entre friable y muy friable, y la resistencia a la penetración indica condiciones de ligera compactación en el horizonte A2 $\left(2,1 \mathrm{~kg} \mathrm{~cm}^{-2}\right)$, producto del prolongado uso anterior agropecuario, en el resto del perfil varió de 0,9 a $1,5 \mathrm{~kg} \mathrm{~cm}^{-2}$. El drenaje interno fue moderado, la capacidad de agua aprovechable muy elevada (284 mm en un metro de profundidad) y la densidad aparente baja $\left(0,61\right.$ a $\left.0,74 \mathrm{~g} \mathrm{~cm}^{-3}\right)$. El suelo presentó un arraigamiento denso, principalmente de especies herbáceas, en los primeros $50 \mathrm{~cm}$ de profundidad (85 a 30 raicillas $\mathrm{dm}^{-2}$ ) y fuerte a mediano en los siguientes $50 \mathrm{~cm}$ (20 a 10 raicillas $\mathrm{dm}^{-2}$ ).

En general, el suelo superficial es fuertemente ácido y pobre en elementos nutritivos, pero rico en materia orgánica y nitrógeno total. La disponibilidad de elementos nutritivos muestra un suelo pobre en fósforo y bases, con un alto porcentaje de saturación de aluminio intercambiable y alto contenido de aluminio extraíble, presenta niveles medios de cobre, zinc y azufre, altos niveles de hierro y muy bajo contenido de sodio. El bajo nivel de manganeso extraíble refleja condiciones de un buen drenaje del suelo (cuadro 1).

Plantaciones y vegetación acompañante. Se evaluaron sendos rodales de $P$. massoniana (rodal 464) y $P$. brutia (rodal 461) establecidos en 1999 a un espaciamiento de 2 x 2 m (25 árboles por rodal), con plantas $2 / 0$, en un sitio con uso anterior de agricultura y praderas. Las semillas eran procedentes de China y Estados Unidos, respectivamente. Las mediciones y recolección de muestras se realizaron en mayo de 2003. De cada árbol se obtuvo la altura total, el diámetro de copa y su caracterización de calidad (sanidad y forma). En cada rodal se obtuvieron dos muestras mezcla de acículas de 10 individuos homogéneamente distribuidos: una muestra de acículas del último período vegetativo o acículas nuevas, y una del período anterior o acículas viejas. En el follaje se analizaron nitrógeno (Kjeldahl) y fósforo, potasio, calcio, magnesio, hierro, manganeso, cobre, zinc y boro luego de calcinación y disolución en $\mathrm{HCl} 10 \%$.

La caracterización de la vegetación acompañante se hizo mediante un listado florístico y la estimación ocular de la cobertura para las especies con un porcentaje de participación $\geq 10 \%$. De esta manera se determinó que las especies herbáceas presentes en el área de estudio, que pueden considerarse malezas, son: Prunella vulgaris, Crepis capillaris, Hypochoeris radicata, Festuca arundinacea, Lotus uliginosus, Dactylis glomerata, Rumex acetosella, Taraxacum officinale, Ranunculus repens y, 
Cuadro 1. Características químicas del suelo $(0-20 \mathrm{~cm})$. Chemical characteristics of the soil $(0-20 \mathrm{~cm})$.

\begin{tabular}{|c|c|c|}
\hline Características & Nivel & Categoría \\
\hline $\mathrm{pH} \mathrm{H}_{2} \mathrm{O}$ & 5,11 & Fuertemente ácido \\
\hline $\mathrm{pH} \mathrm{KCl}$ & 4,78 & Fuertemente ácido \\
\hline $\mathrm{C}$ total $(\%)$ & 8,49 & Alto \\
\hline $\mathrm{N}$ total $(\%)$ & 0,58 & Alto \\
\hline $\mathrm{C} / \mathrm{N}$ & 14,7 & Bajo \\
\hline $\mathrm{P}$ disponible Olsen $\left(\mathrm{mg} \mathrm{kg}^{-1}\right)$ & 1,7 & Muy bajo \\
\hline $\mathrm{K}$ extraíble $\left(\mathrm{mg} \mathrm{kg}^{-1}\right)$ & 57 & Bajo \\
\hline Ca extraíble $\left(\mathrm{mg} \mathrm{kg}^{-1}\right)$ & 156 & Bajo \\
\hline $\mathrm{Mg}$ extraíble $\left(\mathrm{mg} \mathrm{kg}^{-1}\right)$ & 39 & Bajo \\
\hline $\mathrm{Na}$ extraíble $\left(\mathrm{mg} \mathrm{kg}^{-1}\right)$ & 46 & Muy bajo \\
\hline $\mathrm{S}$ disponible $\left(\mathrm{mg} \mathrm{kg}^{-1}\right)$ & 10 & Medio \\
\hline Fe extraíble $\left(\mathrm{mg} \mathrm{kg}^{-1}\right)$ & 121 & Alto \\
\hline Mn extraíble (mg kg-1) & 9 & Bajo \\
\hline $\mathrm{Cu}$ extraíble $\left(\mathrm{mg} \mathrm{kg}^{-1}\right)$ & 4,7 & Medio \\
\hline Zn extraíble $\left(\mathrm{mg} \mathrm{kg}^{-1}\right)$ & 1,3 & Medio \\
\hline B soluble $\left(\mathrm{mg} \mathrm{kg}^{-1}\right)$ & 0,3 & Bajo \\
\hline $\mathrm{Al}$ extraíble $\left(\mathrm{mg} \mathrm{kg}^{-1}\right)$ & 1.690 & Alto \\
\hline Suma Bases $\left(\mathrm{cmol}+\mathrm{kg}^{-1}\right)$ & 1,45 & Muy bajo \\
\hline $\mathrm{Al}$ de intercambio $\left(\mathrm{cmol}+\mathrm{kg}^{-1}\right)$ & 0,36 & Medio \\
\hline Saturación Al (\%) & 19,9 & Alto \\
\hline
\end{tabular}

las arbustivas: Rubus constrictus y Ulex europeaus. La cobertura de malezas fue del $100 \%$ y el tamaño de las plantas herbáceas alcanzó cerca de un metro altura. Las especies arbustivas presentaron baja cobertura $(<10 \%)$ y su altura fue controlada con limpias manuales periódicas (corta manual de vegetación arbustiva).

\section{RESULTADOS}

La sobrevivencia fue de $100 \%$ en ambas especies. El crecimiento medio anual, durante los 4 años de establecimiento, en $P$. massoniana alcanzó a $75 \mathrm{~cm}_{\text {año }}{ }^{-1}$ en altura y $38 \mathrm{~cm}$ año $0^{-1}$ en diámetro de copa; para $P$. brutia estos valores fueron de 48 y $25 \mathrm{~cm}$ año ${ }^{-1}$, respectivamente. Los coeficientes de variación de la altura total y del diámetro de copa fueron levemente mayores en $P$. brutia (10,6 y $16,5 \%$, respectivamente) que en $P$. massoniana (9,2 y $13,1 \%$, respectivamente). La cobertura del suelo por las copas de los árboles alcanzó a $45 \%$ en $P$. massoniana y a $20 \%$ en $P$. brutia.

En consideración a los niveles de referencia foliar para $P$. radiata (Will 1985), P. massoniana estaría bien abastecido de nitrógeno, calcio, magnesio, hierro, manganeso y boro; presentaría algún grado de insuficiencia de fósforo, potasio, cobre y zinc. La alta relación N/P en el follaje de esta especie es indicadora de insuficiencia de fósforo. Por otra parte, $P$. brutia presentaría un contenido satisfactorio en todos los elementos con la excepción de fósforo y potasio, en los cuales tendría alguna insuficiencia de menor grado que en la otra especie (cuadro 2).
Pinus massoniana presentó copa y tronco con un aspecto saludable, no observándose daño mecánico ni por agentes bióticos aunque la mayoría de los fustes (70\%) pesentó torceduras. No se observó presencia de polilla del brote (Rhyacionia bouliana) ni tampoco de hongos foliares, no obstante que Gibson (1979) clasifica a esta especie como moderadamente susceptible al ataque de Dothistroma septospora. Las acículas se encontraban hasta la base del árbol (90\% de copa viva) con edades de 1 a 3 años. Las acículas nuevas presentaron un amarillamiento apical en aproximadamente $3 \mathrm{~mm}$ con un límite definido hacia el verde; en el resto de la acícula no se observaron problemas de malformación ni sanitarios. En las acículas antiguas dicho amarillamiento aumentó hasta los $5 \mathrm{~mm}$, e incluso en algunas de ellas la punta se encontraba con necrosis. Además, sólo en el haz de las acículas se presentaron puntos amarillos. Las acículas más jóvenes eran más cortas respecto a las adultas, pero sin problemas de malformación.

En cambio, $P$. brutia presentó un estado sanitario deficiente, ya que casi la totalidad de las acículas del tercio inferior de la copa estaban muertas o habían caído y también hubo pérdida de acículas en el tercio medio de la copa ( $40 \%$ de copa viva). Se conservaban acículas del último período vegetativo y parcialmente del anterior. Las acículas nuevas presentaban un amarillamiento en sus puntas y lanilla en la base, característica típica del ataque del pulgón lanígero perteneciente a la familia Adelgidae (Pineus borneri) (Gara et al. 1980). Las acículas antiguas estaban completamente necrosadas, con manchas asociadas a Dothistroma septospora y también presentaron lanilla blanca, la cual indica el ataque de pulgones en años anteriores. El porcentaje de ataque de los pulgones alcanzó el $90 \%$ de los árboles y con una intensidad de ataque superior al $40 \%$ de la copa.

Las acículas de $P$. brutia presentaban un aspecto poco vigoroso y torcidas en todo el árbol. Sin embargo, al parecer la torcedura de las acículas se puede deber a características propias de la especie. Llama la atención en estos árboles el pequeño tamaño de sus acículas $(9 \mathrm{~cm}$ de largo), si se compara con los 13 a $16 \mathrm{~cm}$ de largo en su lugar de origen. Las ramas y el tronco en general no presentaron daños mecánicos ni otros daños bióticos.

\section{DISCUSIÓN}

El rendimiento alcanzado por $P$. massoniana equivale a un índice de sitio de 21 a $23 \mathrm{~m}$ si se le aplican los criterios de evaluación para $P$. radiata ${ }^{3}$. En cambio,

3 Alicia Ortega: Instituto de Manejo Forestal, Universidad Austral de Chile (Simulador Radiata, versión docente, 1997), comunicación personal. 
Cuadro 2. Contenido de elementos nutritivos en el follaje de Pinus massoniana y P. brutia, y valores referenciales satisfactorios para $P$. radiata.

Nutrient element contents in the foliage of Pinus massoniana and P. brutia, and satisfactory referential values for $P$. radiata.

\begin{tabular}{|c|c|c|c|c|c|}
\hline \multirow{2}{*}{ Elemento } & \multirow{2}{*}{$\begin{array}{c}\text { Nivel } \\
\text { satisfactorio en } \\
\text { P. radiata* }\end{array}$} & \multicolumn{2}{|c|}{ Pinus massoniana } & \multicolumn{2}{|c|}{ Pinus brutia } \\
\hline & & Hojas nuevas & Hojas viejas & Hojas nuevas & Hojas viejas \\
\hline \multicolumn{6}{|c|}{ Elementos mayores (\%) } \\
\hline Nitrógeno & $>1,5$ & 1,78 & 1,58 & 1,48 & 1,87 \\
\hline Fósforo & $>0,14$ & 0,11 & 0,09 & 0,12 & 0,12 \\
\hline Potasio & $>0,50$ & 0,4 & 0,36 & 0,39 & 0,38 \\
\hline Calcio & $>0,10$ & 0,31 & 0,59 & 0,36 & 0,39 \\
\hline Magnesio & $>0,10$ & 0,14 & 0,09 & 0,14 & 0,1 \\
\hline Relación N/P & $8-12$ & 16,2 & 17,6 & 12,3 & 15,6 \\
\hline \multicolumn{6}{|c|}{ Elementos menores (mg kg-1) } \\
\hline Hierro & $>40$ & 73 & 101 & 55 & 106 \\
\hline Manganeso & $>20$ & 150 & 361 & 183 & 236 \\
\hline Cobre & $>4$ & 3,3 & 2,4 & 4,5 & 4,2 \\
\hline Zinc & $>20$ & 16 & 14 & 42 & 31 \\
\hline Boro & $>12$ & 23 & 25 & 13 & 13 \\
\hline
\end{tabular}

* Will (1985).

P. brutia alcanzaría un índice de sitio de 15 a $18 \mathrm{~m}$. Ello indica que $P$. massoniana se encuentra creciendo a un ritmo comparable con un sitio de calidad baja a media para $P$. radiata; pero $P$. brutia muestra un desarrollo equivalente a un muy mal sitio para $P$. radiata. Este rendimiento en ambas especies se explica, fundamentalmente, a través de la secuencia típica de importancia de los factores del crecimiento de los bosques (Schlatter y Gerding 1995), es decir, macroclima, microclima, propiedades físicas del suelo y propiedades químicas del suelo. Las condiciones climáticas en el arboreto son parecidas a las del lugar de origen de P. massoniana, aunque es más lluvioso en esta área del sur de Chile. Por ello esta especie presentaría una adecuada adaptación a este factor del sitio. Por el contrario, existen grandes diferencias climáticas entre el lugar de origen de $P$. brutia y el arboreto, reflejadas en las condiciones extremas donde esta especie se desarrolla en forma natural, con inviernos muy fríos, extensos períodos secos y baja humedad relativa del aire, lo cual contrasta con las condiciones templadas y húmedas imperantes en el arboreto. En consecuencia, $P$. brutia puede presentar una fuerte incompatibilidad climática en esta zona de Chile.

El suelo del arboreto en el sitio estudiado presenta buenas características de fertilidad en relación con el espacio arraigable (profundidad, estructura) y los regímenes de aire, agua y calor. En cambio, manifiesta limi- taciones de mediana importancia en el régimen de elementos nutritivos. De esta evaluación se desprende que el suelo del arboreto es adecuado para $P$. massoniana, pero presentaría algunas condiciones desfavorables para $P$. brutia, como su acidez y abundante humedad retenida por la textura fina, aunque el suelo no presenta restricciones de drenaje a pesar de la cuantiosa precipitación anual. También puede ser limitante el alto porcentaje de saturación de aluminio que se relaciona con una toxicidad para el desarrollo radicular, que es muy poco probable de encontrar en los suelos de la región originaria de P. brutia.

Ambas plantaciones se han desarrollado en un ambiente de malezas herbáceas, principalmente las que pudieron haber mermado su potencial de crecimiento en los primeros años, como es frecuente en plantaciones forestales en esta región. Cerca de las plantaciones (distante $30 \mathrm{~m}$ aproximadamente) existe un remanente de bosque nativo secundario (renoval) de 15 a $20 \mathrm{~m}$ de altura, constituido principalmente por Nothofagus dombeyi, Nothofagus obliqua, Aextoxicum punctatum y la especie exótica Acacia melanoxylon. Este bosque se encuentra ubicado en la ladera media y baja, formando una protección a las plantaciones de los vientos del noroeste. Sin embargo, dadas las características climáticas originales donde se desarrollan P. brutia y P. massoniana no se espera que este abrigo pueda producir 
una influencia sobre el crecimiento de estas especies. En ningún caso el bosque circundante le provoca sombra a las plantaciones.

Las diferencias de contenidos de elementos nutritivos entre hojas nuevas y viejas muestra en $P$. massoniana una movilidad típica de nitrógeno, fósforo y potasio. Sin embargo, este fenómeno no es evidente en $P$. brutia, especie en la cual se observa un comportamiento atípico con un contenido de nitrógeno en las hojas viejas mayor que en las hojas nuevas. Esto puede explicarse, por una parte, debido a la caída prematura de acículas que impide una adecuada reubicación de los elementos móviles; por otra parte, puede deberse a la incompatibilidad climática que estaría alterando la distribución común de este elemento dentro de la planta, impidiendo su movilidad o haciéndola más lenta. Una situación similar se observó para el nitrógeno en acículas de $P$. cembra de 27 años creciendo en un sitio similar del arboreto ${ }^{4}$, en consecuencia que el clima en su región de origen es de carácter alto alpino.

En las acículas de ambas especies el fósforo presentó un nivel marginal, pero cercano al límite satisfactorio reconocido para $P$. radiata (Will 1985), reflejando una eficiencia de estas especies de Pinus en la absorción de este elemento en un suelo de baja disponibilidad de fósforo. De igual forma, los altos contenidos de calcio y magnesio evidencian la eficiencia de estas especies en la captación de nutrientes desde un suelo pobre, lo que es común en especies pioneras, especialmente si se desarrollan originalmente en suelos muy poco evolucionados. Los síntomas visuales del follaje en ambas especias corresponden principalmente a deficiencias leves de fósforo y potasio, coincidiendo con el análisis foliar. La plantación de $P$. massoniana presentó una mayor demanda nutricional que la de $P$. brutia debido a su mayor tasa de crecimiento; por ello se explica su relativo menor nivel nutricional en el follaje. En cambio, $P$. brutia, con su menor tasa de crecimiento, presentó contenidos satisfactorios en la mayoría de los elementos mayores y en todos los elementos menores. En consecuencia, el escaso desarrollo de los árboles de esta especie no se debería a deficiencias nutricionales, sino que a condiciones climáticas desfavorables y a problemas sanitarios por agentes bióticos.

El estado sanitario difiere entre los dos rodales. $\mathrm{Pi}$ nus brutia presentó condiciones de menor calidad sanitaria, con el $100 \%$ de los árboles de baja vitalidad en un estado de degeneración, según la clasificación de Roloff (1993), con copas abiertas y translúcidas debido a la escasa formación de ramas secundarias y a la abundante

4 Laboratorio de Nutrición y Suelos Forestales, Universidad Austral de Chile; datos no publicados. caída prematura de acículas. Esta situación estaría indicando las limitaciones de $P$. brutia en esta área de introducción. En cambio, $P$. massoniana manifestó un estado de exploración, con copas en plena capacidad de crecimiento.

\section{CONCLUSIONES}

Pinus massoniana manifestó una adecuada compatibilidad climática y edáfica en el sitio del arboreto; presentó moderadas expectativas de crecimiento, sin evidencias actuales de daños entomopatológicos, pero con deficiencias leves de fósforo y potasio coincidentes con la baja oferta del suelo.

Pinus brutia mostró incompatibilidad climática que proyecta malas expectativas de crecimiento, aunque no demuestra incompatibilidad edáfica en el sitio del arboreto. Los árboles expresaron deficiencia leve de fósforo y potasio. Esta especie exhibió un estado sanitario malo, por un alto ataque de pulgón lanígero (Pineus borneri) y del hongo desfoliador de la banda roja (Dothistroma septospora).

\section{REFERENCIAS}

CIREN (Centro de Información de Recursos Naturales, CL). 1999. Descripciones de suelos. Materiales y símbolos. Estudio agrológico de la provincia de Valdivia, X Región, Chile. Santiago. 199 p.

Düsterhöft H. 2003. Biomasse eines Mischwaldbestandes in Valdivia, Chile. Tesis Forestal. Göttingen, Alemania. Facultad de Manejo de Recursos Forestales. Universidad Georg-August. 72 p.

Fuenzalida H. 1965. Clima. In Corporación de Fomento de la Producción (CORFO). Geografía Económica de Chile. Texto Refundido. Santiago. p. 99-152.

Gara R, L Cerda, M Donoso. 1980. Manual de entomología forestal. Valdivia, Chile, Facultad de Ingeniería Forestal, Universidad Austral de Chile. 61 p.

Gerding V, JE Schlatter. 1995. Variables y Factores del sitio de importancia para la productividad de Pinus radiata D. Don en Chile. Bosque 16(2): 39-56.

Gibson AS. 1979. Diseases of forest trees widely planted as exotics in the tropics and southern hemisphere. Part II. The Genus Pinus. Kew, Inglaterra. Commonwealth Mycological Institute. $130 \mathrm{p}$.

Huber A. 1970. Diez años de observaciones climatológicas en la estación Teja-Valdivia (Chile) 1960-1969. Valdivia, Chile. Universidad Austral de Chile. 60 p.

Huber H. 1995. El arboretum de la Universidad Austral de Chile, área de investigación y educación forestal. Tesis Ingeniero Forestal. Valdivia, Chile. Facultad de Ciencias Forestales, Universidad Austral de Chile. 54 p.

Roloff A. 1993. Kronenentwicklung und Vitalitätsbeurteilung ausgewählter Baumarten der gemäbigten Breiten. Göttin- 
gen, Alemania. Schriften aus der Forstlichen Fakultät der Universität Göttingen und der Niederdersachsischen Forstlichen Versuchsanstalt. Band 93. 258 p.

Schlatter JE, V Gerding. 1995. Método de clasificación de sitios para la producción forestal, ejemplo en Chile. Bosque 16(2): 13-20.

Schlatter JE, V Gerding, H Huber. 1995. Sistema de ordenamiento de la tierra, herramienta para la panificación forestal aplicada a la X Región. Valdivia, Chile. Serie Técnica, Universidad Austral de Chile. 93 p.
Schlatter JE, R Grez, V Gerding. 2003. Manual para el reconocimiento de suelos. Valdivia, Chile. Universidad Austral de Chile. 114 p.

Schlegel F. 1985. Arboretum. Valdivia, Chile. Universidad Austral de Chile, sp.

Vidaković M. 1991. Conifers. Morphology and variation. Zagreb. Grafički Zavod Hrvatske. 754 p.

Will G. 1985. Nutrient deficiencies and fertiliser use in New Zealand exotic forests. Rotorua, Nueva Zelandia. FRI Bulletin № 97.53 p.

Recibido: 10.12 .03

Aceptado: 09.03.06 